\title{
Magnetic ordering via itinerant ferromagnetism in a metal-organic framework
}

\author{
Jesse G. Park ${ }^{1}$, Brianna A. Collins² ${ }^{2}$ Lucy E. Darago1, Tomče Runčevski³ ${ }^{3}$, \\ Michael L. Aubrey ${ }^{1}$, Henry Z. H. Jiang ${ }^{1,4}$, Ever Velasquez ${ }^{5}$, Mark A. Green ${ }^{6}$, \\ Jason D. Goodpastor ${ }^{2}$ \& Jeffrey R. Long ${ }^{1,4,5 *}$
}

${ }^{1}$ Department of Chemistry, University of California, Berkeley, California 94720, USA.

${ }^{2}$ Department of Chemistry, University of Minnesota - Twin Cities, Minneapolis, Minnesota 55455, USA.

${ }^{3}$ Department of Chemistry, Southern Methodist University, Texas 75205, USA.

${ }^{4}$ Materials Sciences Division, Lawrence Berkeley National Laboratory, Berkeley, California 94720, USA.

${ }^{5}$ Department of Chemical and Biomolecular Engineering, University of California, Berkeley, California 94720, USA.

${ }^{6}$ School of Physical Sciences, University of Kent, Canterbury, Kent CT2 7NH, United Kingdom.

*Correspondence to: jrlong@ berkeley.edu (J.R.L.) 
Materials that combine magnetic order with other desirable physical attributes offer to revolutionize our energy landscape. Indeed, such materials could find transformative applications in spintronics, quantum sensing, low-density magnets, and gas separations. ${ }^{1-3}$ As a result, efforts to design multifunctional magnetic materials have recently moved beyond traditional solid-state materials to metal-organic solids. Among these, metal-organic frameworks in particular bear structures that offer intrinsic porosity, vast chemical and structural programmability, and tunability of electronic properties. ${ }^{4}$ Nevertheless, magnetic order within metal-organic frameworks has generally been limited to low temperatures, owing largely to challenges in creating strong magnetic exchange in extended metal-organic solids. ${ }^{3}$ Here, we employ the phenomenon of itinerant ferromagnetism to realize magnetic ordering at $T_{\mathrm{C}}=225 \mathrm{~K}$ in a mixed-valence chromium(II/III) triazolate compound, representing the highest ferromagnetic ordering temperature yet observed in a metalorganic framework. The itinerant ferromagnetism is shown to proceed via a doubleexchange mechanism, the first such observation in any metal-organic material. Critically, this mechanism results in variable-temperature conductivity with barrierless charge transport below $T_{\mathrm{C}}$ and a large negative magnetoresistance of $23 \%$ at $5 \mathrm{~K}$. These observations suggest applications for double-exchange-based coordination solids in the emergent fields of magnetoelectrics and spintronics. ${ }^{5,6}$ Taken together, the insights gleaned from these results are expected to provide a blueprint for the design and synthesis of porous materials with synergistic high-temperature magnetic and charge transport properties.

The development of multifunctional magnets with optimized magnetic properties in concert with other physical properties such as porosity and high magnetoresistance remains a daunting challenge, with promise to enable new applications in green technologies ${ }^{7}$ and next-generation data processing and storage. ${ }^{6}$ For realization of commercial applications, it is crucial for a magnet to exhibit a high magnetic ordering temperature beyond potentially elevated operating temperatures. The vast majority of solid-state permanent magnets with high ordering temperatures are based on itinerant magnetism. ${ }^{8}$ In particular, a specific form of itinerant magnetism, known as a double-exchange mechanism, was discovered by Zener in 1951 to explain the coexistence of metallic conductivity and high-temperature ferromagnetism in perovskite mixed-valence manganites, which contain $\mathrm{Mn}^{\mathrm{III}}$ and $\mathrm{Mn}^{\mathrm{IV}}$ ions connected by bridging $\mathrm{O}^{2-}$ ions. ${ }^{9}$ Here, an itinerant electron with an oriented spin is delocalized between $e_{\mathrm{g}}$ orbitals of neighboring $\mathrm{Mn}$ ions and consequently promotes a parallel alignment of spins for the localized electrons in $t_{2 g}$ orbitals, in accordance with Hund's rules. Many such double-exchange solid-state materials, including cobaltites and Heusler alloys, have since been discovered and investigated for applications in spintronics devices. ${ }^{6,10,11}$

As an alternative to traditional solid-state materials, metal-organic frameworks, a subclass of coordination solids, are composed of inorganic building units connected by polytopic organic linkers. Compared to solid-state materials, metal-organic frameworks can offer tremendous synthetic versatility for fine-tuning their chemical and physical properties. For instance, organic linkers with predictable binding modes can be utilized to yield frameworks with unique crystal 
structures and physical properties, which are easily modified through methods including substitution of electron donating/withdrawing substituents on the ligand, post-synthetic redox chemistry, and metal or linker exchange. ${ }^{4,12}$ Furthermore, a combination of a long-range magnetic order and porosity in framework materials could lead to the realization of lightweight permanent magnets and magnetic separation media., ${ }^{3,13}$ Nevertheless, the overwhelming majority of framework materials are not permanent magnets, owing primarily to the inability of diamagnetic organic linkers to mediate the strong magnetic coupling requisite for long-range order. Indeed, only a few strategies have been developed to synthesize coordination solids with high magnetic ordering temperatures, including the employment of short diamagnetic inorganic ligands or organic radical ligands, as exemplified by Prussian blue analogues and the amorphous material V(tetracyanoethylene) $\sim 2$, respectively. ${ }^{3,14,15}$ Alternatively, a potentially powerful, yet unrealized strategy involves the utilization of itinerant charge carriers via a double exchange mechanism. In addition to achieving high ordering temperatures in metal-organic magnets containing diamagnetic linkers, this approach may further provide a means of introducing metallic electronic conductivity.

While double exchange is a recognized phenomenon in solid-state materials, ${ }^{9-11}$ examples in coordination solids with organic ligands are limited to molecular compounds. ${ }^{16,17}$ Azolate ligands have strong $\sigma$-donating and $\pi$-accepting abilities, and when coordinated to octahedral metal ions with diffuse $d_{\pi}$ orbitals of favorable energies, strong $\pi$ - $d$ conjugation between ligand and metal orbitals may arise. ${ }^{16,18,19}$ Furthermore, compact, symmetrical azolate ligands can support crystal structures consisting of infinite metal-azolate chains with short metal-metal distances and an octahedral coordination environment around metal ions, providing efficient pathways for longrange charge transport and magnetic interaction. ${ }^{20,21,22}$ Herein, we report the mixed-valence framework material $\mathrm{Cr}(\operatorname{tri})_{2}\left(\mathrm{CF}_{3} \mathrm{SO}_{3}\right)_{0.33}$, which exhibits itinerant ferromagnetism with $T_{\mathrm{C}}=225$ $\mathrm{K}$ via a double-exchange mechanism.

The compound $\mathrm{Cr}(\operatorname{tri})_{2}\left(\mathrm{CF}_{3} \mathrm{SO}_{3}\right)_{0.33}$ is formed through a solvothermal reaction between anhydrous $\mathrm{Cr}\left(\mathrm{CF}_{3} \mathrm{SO}_{3}\right)_{2}$ and $1 \mathrm{H}$-1,2,3-triazole in $\mathrm{N}, \mathrm{N}$-dimethylformamide (see Methods and Supplementary Information). The resulting purple solid consists of octahedron-shaped crystals with an edge dimension of $\sim 0.5 \mu \mathrm{m}$ (Extended Data Fig. 1) and is structurally analogous to reported M(tri) 2 (M $=\mathrm{Mg}, \mathrm{Mn}, \mathrm{Fe}, \mathrm{Co}, \mathrm{Cu}, \mathrm{Zn}, \mathrm{Cd})$ materials. $^{21,23,24}$ The material has a diamondoid-type structure formed by corner-sharing tetrahedral pentanuclear repeating units (Fig. 1a), where each unit comprises two crystallographically distinct $\mathrm{Cr}$ ions bridged by a 1,2,3-triazolate ligand with $\mathrm{Cr} 1$ $\mathrm{N} 1$ and Cr2-N2 distances of 2.067(4) and 2.031(6) Å, respectively (Fig. 1b). In addition, the pores of the framework are occupied by disordered $\mathrm{CF}_{3} \mathrm{SO}_{3}{ }^{-}$ions. A careful analysis of the refined chemical occupancies reveals the presence of $0.34 \mathrm{CF}_{3} \mathrm{SO}_{3}{ }^{-}$ions per $\mathrm{Cr}$ ion, which is close to the expected chemical formula of $\mathrm{Cr}(\mathrm{tri})_{2}\left(\mathrm{CF}_{3} \mathrm{SO}_{3}\right)_{0.33}$ if each pore within the framework is filled with a single $\mathrm{CF}_{3} \mathrm{SO}_{3}{ }^{-}$ion. The presence of inserted $\mathrm{CF}_{3} \mathrm{SO}_{3}{ }^{-}$ions was further confirmed by the low BET surface area of $80 \mathrm{~m}^{2} \mathrm{~g}^{-1}$ calculated from an $\mathrm{N}_{2}$ adsorption isotherm (Extended Data Fig. 3) and elemental analysis for $\mathrm{C}, \mathrm{H}$, and $\mathrm{N}$ that is consistent with the formula $\mathrm{Cr}(\operatorname{tri})_{2}\left(\mathrm{CF}_{3} \mathrm{SO}_{3}\right)_{0.33}$ (see Methods). The presence of charge-balancing $\mathrm{CF}_{3} \mathrm{SO}_{3}{ }^{-}$ions in the pores of the framework indicates that the material contains mixed-valence $\mathrm{Cr}^{\mathrm{II} / \mathrm{III}}$ centers (see Supplementary Information). 
A diffuse reflectance UV-vis-near-IR spectrum of $\mathrm{Cr}(\operatorname{tri})_{2}\left(\mathrm{CF}_{3} \mathrm{SO}_{3}\right)_{0.33}$ was obtained to confirm and further probe the mixed-valence electronic structure (Fig. 2a). Notably, the spectrum exhibits an intense absorption band in the near-IR region between 5,000 and 12,000 $\mathrm{cm}^{-1}$, with the absorbance increasing continuously to lower energies. We assign the observed band to an intervalence charge-transfer transition between mixed-valence $\mathrm{Cr}^{\mathrm{II} / \mathrm{III}}$ centers. Furthermore, the IR spectrum of $\mathrm{Cr}(\mathrm{tri})_{2}\left(\mathrm{CF}_{3} \mathrm{SO}_{3}\right)_{0.33}$ exhibits a broad absorption between 1,500 and $6,000 \mathrm{~cm}^{-1}$, owing to extension of the intervalence charge-transfer transition band into the mid-IR region (Fig. 2b). The absorption band masks most of the expected vibrational modes, leaving few detectable, sharp features. Indeed, absorption bands of similar energies have also been reported for other mixedvalence coordination solids and charge transfer salts. ${ }^{19,21,25,26}$ We note that the presence of such low-energy transitions suggests a negligible optical band gap stemming from a near-continuous distribution of mid-gap states.

Variable-temperature dc magnetic susceptibility measurements were performed to investigate the magnetic properties of $\mathrm{Cr}(\mathrm{tri})_{2}\left(\mathrm{CF}_{3} \mathrm{SO}_{3}\right)_{0.33}$, (Fig. 3a). The plot of magnetization versus temperature features a gradual increase in magnetization upon decreasing the temperature from $350 \mathrm{~K}$, followed by a sudden increase below $\sim 250 \mathrm{~K}$. This sharp rise indicates a magnetic phase transition accompanied by an increased magnetic correlation length within the system. The plot exhibits bifurcation of field-cooled and zero-field-cooled curves below the transition temperature, indicative of spontaneous magnetization. Furthermore, a Curie-Weiss fit to the inverse dc susceptibility data from 300 to $350 \mathrm{~K}$ affords $\theta_{\mathrm{CW}}=268 \mathrm{~K}$, with the positive Curie-Weiss temperature suggesting dominant ferromagnetism, consistent with double exchange (see Supplementary Information and Extended Data Fig. 4a). The presence of magnetic order was confirmed by ac magnetic susceptibility measurements (Extended Data Fig. 4b-f), which show sharp increases in both the in-phase $\left(\chi_{\mathrm{M}}{ }^{\prime}\right)$ and the out-of-phase $\left(\chi_{\mathrm{M}}{ }^{\prime \prime}\right)$ susceptibilities below $250 \mathrm{~K}$. A precise magnetic ordering temperature was determined as $T_{\mathrm{C}}=225 \mathrm{~K}$ by locating the temperature at which $\chi_{\mathrm{M}}$ " undergoes a sharp increase from zero. To the best of our knowledge, $\mathrm{Cr}(\text { tri })_{2}\left(\mathrm{CF}_{3} \mathrm{SO}_{3}\right)_{0.33}$ exhibits the highest ferromagnetic ordering temperature among all coordination solids and the highest temperature for any type of magnetic order among structurally characterized metal-organic frameworks, with the previous records being $T_{\mathrm{C}}=107 \mathrm{~K}$ for $\left[\left\{\mathrm{Ru}_{2}(\mathrm{~m}\right.\right.$ fluorobenzoate $\left.)_{2}\right\}_{2}$ (BTDA-TCNQ) $\quad$ (BTDA-TCNQ = bis $(1,2,5-$ thiadiazolo)tetracyanoquinodimethane) and $T_{\mathrm{N}}=171 \mathrm{~K}$ for $\mathrm{Mn}(\mathrm{TCNE})_{3 / 2}\left(\mathrm{I}_{3}\right)_{1 / 2}(\mathrm{TCNE}=$ tetracyanoethylene), respectively. ${ }^{3,27,28}$ The variable-field magnetization data collected between 3 and $270 \mathrm{~K}$ exhibit an S-shaped curve that quickly saturates at a magnetization of $2.39 \mu_{\mathrm{B} \mathrm{mol}}^{-1}$ at $3 \mathrm{~K}$, close to the expected value of $2.33 \mu_{\mathrm{B}} \mathrm{mol}^{-1}$ for ferromagnetically coupled low-spin $\mathrm{Cr}^{\mathrm{II}}$ and $\mathrm{Cr}^{\mathrm{III}}$ centers present in a 2:1 ratio (Fig. 3b). The magnetization data do not exhibit a hysteresis, with a negligible remnant magnetization at all temperatures, indicating that $\mathrm{Cr}(\operatorname{tri})_{2}\left(\mathrm{CF}_{3} \mathrm{SO}_{3}\right)_{0.33}$ is a soft magnet. The absence of hysteresis is most likely due to the cubic crystal structure of the compound, which results in a clear lack of bulk magnetocrystalline anisotropy.

Insights into the electronic structure of $\mathrm{Cr}(\operatorname{tri})_{2}\left(\mathrm{CF}_{3} \mathrm{SO}_{3}\right)_{0.33}$ were obtained using Density Functional Theory (DFT) (see Supplementary Information for full computational details). Calculations were performed on the full three-dimensional unit cell, which contains $24 \mathrm{Cr}$ atoms, 
48 triazolate ligands, and 8 triflate anions. The results suggest that the ferromagnetic state is lower in energy compared to the antiferromagnetic state by $0.127 \mathrm{eV} / \mathrm{Cr}$ atom, in agreement with the magnetic measurements. In the ferromagnetic state, the calculated saturation magnetic moment is $2.41 \mu_{\mathrm{B}}$, which is in excellent agreement with the experimentally measured value of $2.39 \mu_{\mathrm{B}}$. The band structure of $\mathrm{Cr}(\text { tri })_{2}\left(\mathrm{CF}_{3} \mathrm{SO}_{3}\right)_{0.33}$ (Fig. 1a) displays highly dense, spin-polarized bands near the Fermi energy with a band dispersion of $\sim 1 \mathrm{eV}$. The projected band structure (Fig. 1b) and projected density of states (Fig. 1d) show that both $\mathrm{Cr} d$ orbitals and $\mathrm{N} p$ orbitals of the triazolate ligands contribute to the valence band maximum and the conduction band minimum. Near the Fermi energy, the frontier orbitals of the bands are dominated by the $\mathrm{Cr} d_{\pi}$ orbitals and $\mathrm{N} p$ orbitals, with approximately $32 \%$ hybridization between them, indicating a strong hybridization between the $\mathrm{Cr} d$ and $\mathrm{N} p$ orbitals and $\pi-d$ conjugation. The localized charge density (Extended Data Fig. 9) shows alternating high and low charge $\mathrm{Cr}$ centers. The $\mathrm{Cr}$ centers of lower and higher charges exhibit lower and higher magnetic moments, respectively, consistent with the mixed-valence lowspin $\mathrm{Cr}^{\mathrm{II} / \mathrm{III}}$ centers. Notably, the charge density and projected density of states both indicate strong spin-polarization. Taken together, these computational results demonstrate a ferromagnetic ground state for $\mathrm{Cr}(\operatorname{tri})_{2}\left(\mathrm{CF}_{3} \mathrm{SO}_{3}\right)_{0.33}$, and the high density of spin-polarized bands near the Fermi energy arising from a strong $\pi$ - $d$ hybridization suggests delocalization of electrons in the valence band maximum, consistent with double exchange stemming from the mixed-valence metal centers.

Variable-temperature conductivity measurements were performed to investigate the charge transport properties associated with double exchange in $\mathrm{Cr}(\mathrm{tri})_{2}\left(\mathrm{CF}_{3} \mathrm{SO}_{3}\right)_{0.33}$ (Fig. 4e and Extended Data Fig. 6). Upon cooling from 300 to $200 \mathrm{~K}$, the conductivity decreases very slightly from $\sigma=$ $1.4(1) \times 10^{-2}$ to $1.3(1) \times 10^{-2} \mathrm{~S} \mathrm{~cm}^{-1}$. Despite the presence of a slight hysteresis between data measured upon cooling vs warming the sample, the cooling data above $200 \mathrm{~K}$ could be fit using the three-dimensional variable-range hopping model (see Supplementary Information). Below $T_{\mathrm{C}}$ and down to $80 \mathrm{~K}$, the compound exhibits a conductivity that is nearly temperature-independent, with $\sigma \approx 1.2(1) \times 10^{-2} \mathrm{~S} \mathrm{~cm}^{-1}$. An Arrhenius fit to the data in this temperature range yields a small activation energy of $0.14 \mathrm{meV}$, suggestive of highly delocalized charge carriers. Upon cooling below $80 \mathrm{~K}$, we observed a decrease in conductivity to $\sigma=4.4(1) \times 10^{-3} \mathrm{~S} \mathrm{~cm}^{-1}$ at $5 \mathrm{~K}$. The lowtemperature data are best fit with the Efros-Shklovskii variable-range hopping model, highlighting a transition back to localized small polarons (see Supplementary Information). ${ }^{29}$ Next, conductivity measurements were performed on the same sample pellet while cooling the cell under an applied field of $7 \mathrm{~T}$. Strikingly, the conductivity increases as the temperature is lowered from 225 to $80 \mathrm{~K}$. At $5 \mathrm{~K}, \mathrm{Cr}(\text { tri })_{2}\left(\mathrm{CF}_{3} \mathrm{SO}_{3}\right)_{0.33}$ exhibits a remarkable maximum negative magnetoresistance of $\sim 23 \%$ (Fig. 4f), representing one of the highest values for any coordination solid. ${ }^{30-33}$ While unprecedented in coordination solids, the observed barrier-less transport behavior in conjunction with the large negative magnetoresistance is consistent with a double exchange mechanism in $\mathrm{Cr}(\mathrm{tri})_{2}\left(\mathrm{CF}_{3} \mathrm{SO}_{3}\right)_{0.33}$. The computational results suggest that the partially filled spindown bands near the Fermi level give rise to a current density that is carried mainly by spin-down charge carriers. Due to the spin-polarized current, the presence of any tunneling junctions consisting of ferromagnetic domains/layers can yield large magnetoresistance. As $\mathrm{Cr}(\text { tri })_{2}\left(\mathrm{CF}_{3} \mathrm{SO}_{3}\right)_{0.33}$ is a soft ferromagnet, the material most likely consists of randomly aligned ferromagnetic domains under zero applied magnetic field. Under an applied magnetic field, the 
ferromagnetic domains can align parallel to each other, allowing a facile transport of spinpolarized currents. Indeed, large negative magnetoresistance has commonly been observed in solid-state materials with itinerant ferromagnetism. ${ }^{34,35}$

The foregoing results demonstrate the discovery of itinerant ferromagnetism in a metal-organic framework, which gives rise to an unprecedented magnetic ordering temperature of $T_{\mathrm{C}}=225 \mathrm{~K}$ and remarkably high magnetoresistance. Importantly, the employed synthetic approach of utilizing $\pi-d$ conjugation between organic linkers and mixed-valence metal centers with diffuse $d_{\pi}$ orbitals provides a general blueprint from which to design materials with enhanced properties, given the vast chemical versatility offered by metal-organic frameworks.

\section{References}

1. Miller, J. S. \& Drillon, M. Magnetism: Molecules to Materials II (Wiley-VCH, 2001).

2. Coronado, E., Palacio, F. \& Veciana, J. Molecule-based magnetic materials. Angew. Chem. Int. Ed. 42, 2570-2572 (2003).

3. Dechambenoit, P. \& Long, J. R. Microporous magnets. Chem. Soc. Rev. 40, 3249-3265 (2011).

4. Yaghi, O. M. et al. Reticular synthesis and the design of new materials. Nature 423, 705714 (2003).

5. Tian, Y. et al. Observation of resonant quantum magnetoelectric effect in a multiferroic metal-organic framework. J. Am. Chem. Soc. 138, 782-785 (2016).

6. Felser, C., Fecher, G. H. \& Balke, B. Spintronics: a challenge for materials science and solid-state chemistry. Angew. Chem. Int. Ed. 46, 668-699 (2007).

7. Gutfleicsch, O. et al. Magnetic materials and devices for the $21^{\text {st }}$ century: stronger, lighter and more energy efficient. Adv. Mater. 23, 821-842 (2011).

8. Kübler, J. Theory of itinerant electron magnetism (Oxford Univ. Press, 2000).

9. Zener, C. Interaction between the $d$-shells in the transition metals. II. Ferromagnetic compounds of manganese with perovskite structure. Phys. Rev. 82, 403-405 (1951).

10. Briceño, G., Chang, H., Sun, X., Schultz, P. G. \& Xiang, X.-D. A Class of Cobalt Oxide Magnetoresistance Materials Discovered with Combinatorial Synthesis. Science 270, 273-275 (1995).

11. Şaşığlu, E., Sandratskii, L. M. \& Bruno, P. Role of conduction electrons in mediating exchange interactions in Mn-based Heusler alloys. Phys. Rev. B 77, 064417 (2008).

12. Calbo, J., Golomb, M. J. \& Walsh, A. Redox-active metalo-organic frameworks for energy conversion and storage. J. Mater. Chem. A 7, 16571-16597 (2019).

13. Kosaka, W. et al. Gas-responsive porous magnet distinguishes the electron spin of molecular oxygen. Nat. Commun. 9, 5420 (2018).

14. Ruiz, E., Rodríguez-Fortea, A., Alvarez, S. \& Verdaguer, M. Is it possible to get high $T_{\mathrm{c}}$ magnets with Prussian blue analogues? A theoretical prospect. Chem Eur. J. 11, $2135-$ 2144 (2005).

15. Manriquez, J. M., Yee, G. T., McLean, R. S., Epstein, A. J. \& Miller, J. S. A roomtemperature molecular/organic-based magnet. Science 252, 1415-1417 (1991).

16. Bechlar, B. et al. High-spin ground states via electron delocalization in mixed-valence imidazolate-bridged divanadium complexes. Nat. Chem. 2, 362-368 (2010). 
17. Gaudette, A. I. et al. Electron hopping through double-exchange coupling in a mixedvalence diiminobenzoquinone-bridged Fe2 complex. J. Am. Chem. Soc. 137, 1261712626 (2015).

18. Schulze, B. \& Schubert, U. S. Beyond click chemistry - supramolecular interactions of 1,2,3-triazoles. Chem. Soc. Rev. 43, 2522-2571 (2014).

19. Aubrey, M. L. et al. Electron delocalization and charge mobility as a function of reduction in a metal-organic framework. Nat. Mater. 17, 625-632 (2018).

20. Zhang, J. P., Zhang, Y. B., Lin, J. B. \& Chen, X. M. Metal azolate frameworks: from crystal engineering to functional materials. Chem. Rev. 112, 1001-1033 (2012).

21. Park, J. G. et al. Charge delocalization and bulk electronic conductivity in the mixedvalence metal-organic framework $\mathrm{Fe}(1,2,3 \text {-triazolate })_{2}\left(\mathrm{BF}_{4}\right)_{x} . J$. Am. Chem. Soc. 140, 8526-8534 (2018).

22. Brunschwig, B. S., Creutz, C. \& Sutin, N. Optical transitions of symmetrical mixedvalence systems in the class II-III transition regime. Chem Soc. Rev. 31, 168-184 (2002).

23. Gándara, F. et al. Porous, conductive metal-triazolates and their structural elucidation by the charge-flipping method. Chem. Eur. J. 18, 10595-10601 (2012).

24. Zhou, X.-H., Peng, Y.-H., Du, X.-D., Zuo, J.-L. \& You, X.-Z. Hydrothermal syntheses and structures of three novel coordination polymers assembled from 1,2,3-triazolate ligands. Cryst. Eng. Comm. 11, 1964-1970 (2009).

25. Collman, J. P. et al. Synthetic, electrochemical, optical, and conductivity studies of coordination polymers of iron, ruthenium, and osmium octaethylporphyrin. J. Am. Chem. Soc. 109, 4606-4614 (1987).

26. Tanner, D. B., Jacobsen, C. S., Garito, A. F. \& Heeger, A. J. Infrared studies of the energy gap in tetrathiofulvalene-tetracyanoquinodimethane (TTF-TCNQ). Phys. Rev. B 13, 3381-3404 (1976).

27. Motokawa, N., Miyasaka, H., Yamashita, M. \& Dunbar, K. R. An Electron-Transfer Ferromagnet with $T_{\mathrm{c}}=107 \mathrm{~K}$ Based on a Three-Dimensional $\left[\mathrm{Ru}_{2}\right]_{2} / \mathrm{TCNQ}$ System. Angew. Chem. Int. Ed. 47, 7760-7763 (2008).

28. Stone, K. H. et al. Mn ${ }^{\mathrm{II}}(\mathrm{TCNE})_{3 / 2}\left(\mathrm{I}_{3}\right)_{1 / 2}-\mathrm{A}$ 3D Network-Structured Organic-Based Magnet and Comparison to a 2D Analog. Adv. Mater. 22, 2514-2519 (2010).

29. Efros, A. L. \& Shklovskii, B. I. Coulomb gap and low temperature conductivity of disordered systems. J. Phys. C: Solid State Phys. 8, L49-56 (1975).

30. Raju, N. P. et al. Anomalous magnetoresistance in high-temperature organic-based magnetic semiconducting V(TCNE) $)_{x}$ films. J. Appl. Phys. 93, 6799 (2003).

31. Coronado, E., Prieto-Ruiz, J. P. \& Prima-Garcia, H. Spin polarization in electrodeposited thin films of the molecule-based magnetic semiconductor $\mathrm{Cr}_{5.5}(\mathrm{CN})_{12} \cdot 11.5 \mathrm{H}_{2} \mathrm{O}$. Chem. Commun. 49, 10145-10147 (2013).

32. $\mathrm{Lu}, \mathrm{Y}$. et al. Thin-film deposition of an organic magnet based on vanadium methyl tricyanoethylenecarboxylate. Adv. Mater. 26, 7632-7636 (2014).

33. Black, N. et al. Giant negative magnetoresistance in Ni(quinoline-8-selenoate)2. Phys. Chem. Chem. Phys. 20, 514-519 (2018).

34. Coey, J. M. D. \& Venkatesan, M. Half-metallic ferromagnetism: Example of $\mathrm{CrO}_{2}$. J. Appl. Phys. 91, 8345-8350 (2002)

35. Xiao, J. Q., Jian, J. S. \& Chien, C. L. Giant magnetoresistance in nonmultilayer magnetic systems. Phys. Rev. Lett. 68, 3749-3752 (1992). 
Figures
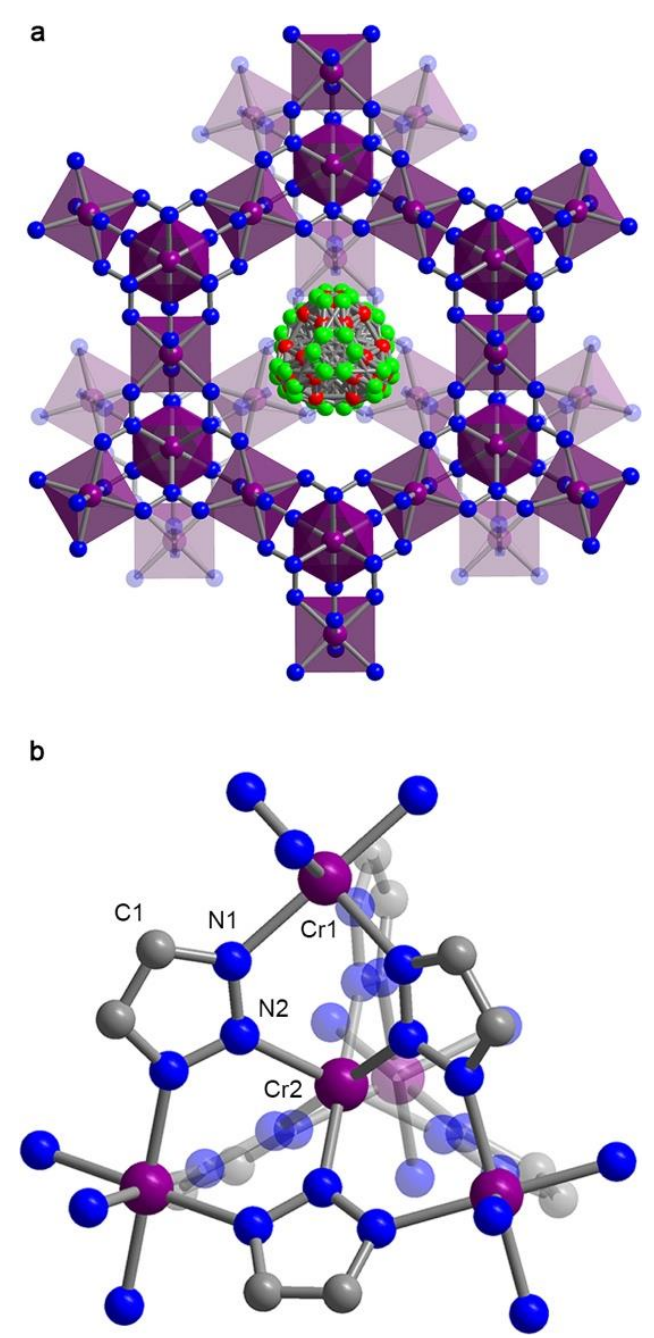

Figure 1 Solid-state structures. a, Portions of the $\mathrm{Cr}-\mathrm{N}$ sublattice of the $\mathrm{Cr}(\operatorname{tri})_{2}\left(\mathrm{CF}_{3} \mathrm{SO}_{3}\right)_{0.33}$ structure determined from analysis of powder X-ray diffraction data collected at $360 \mathrm{~K}$, showing continuous chromium-triazolate chains forming a diamondoid-type lattice and a disordered, charge-balancing trifluoromethanesulfonate anion in the pore cavity. b, A tetrahedral, pentanuclear repeating unit. Purple, yellow, green, red, blue, and grey spheres represent $\mathrm{Cr}, \mathrm{S}, \mathrm{F}, \mathrm{O}, \mathrm{N}$, and C atoms, respectively; $\mathrm{H}$ atoms are omitted for clarity. 

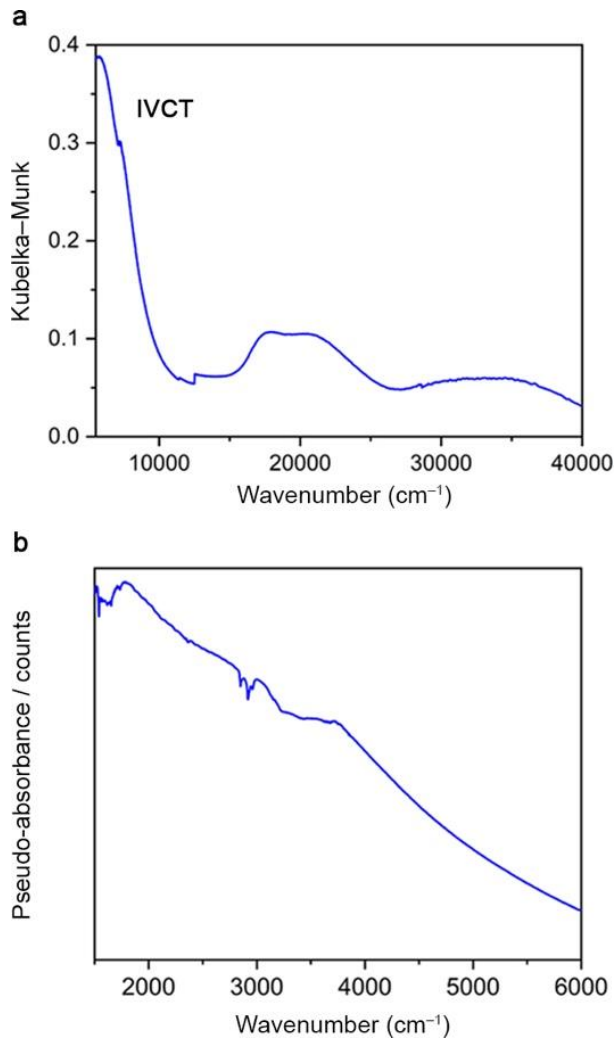

Figure 2 Spectroscopic characterization. a, b, Diffuse reflectance UV-vis-NIR (a) and infrared (b) spectra of $\mathrm{Cr}(\operatorname{tri})_{2}\left(\mathrm{CF}_{3} \mathrm{SO}_{3}\right)_{0.33}$ collected at $300 \mathrm{~K}$.
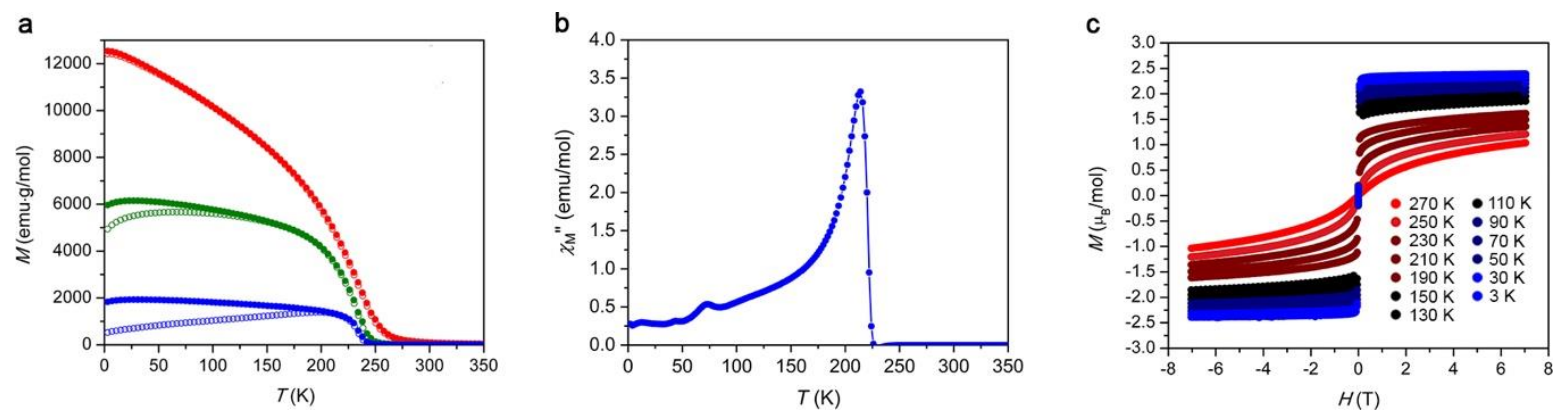

Figure 3 Magnetic properties of $\mathrm{Cr}(\operatorname{tri})_{2}\left(\mathrm{CF}_{3} \mathrm{SO}_{3}\right)_{0.33}$. a, Variable-temperature zero-field-cooled (open circles) and field-cooled (closed circles) magnetic susceptibility data collected at selected dc magnetic fields of 1000 (red), 100 (green), and 25 Oe (blue). b, Variable-temperature out-ofphase ac magnetic susceptibility $\left(\chi^{\prime \prime}\right)$ collected with ac oscillating magnetic field of 4 Oe at a frequency of $10 \mathrm{~Hz}$ and under zero dc magnetic field. c, Variable-field magnetization data collected at selected temperatures with a sweep rate of $33 \mathrm{Oe} \mathrm{s}^{-1}$. 

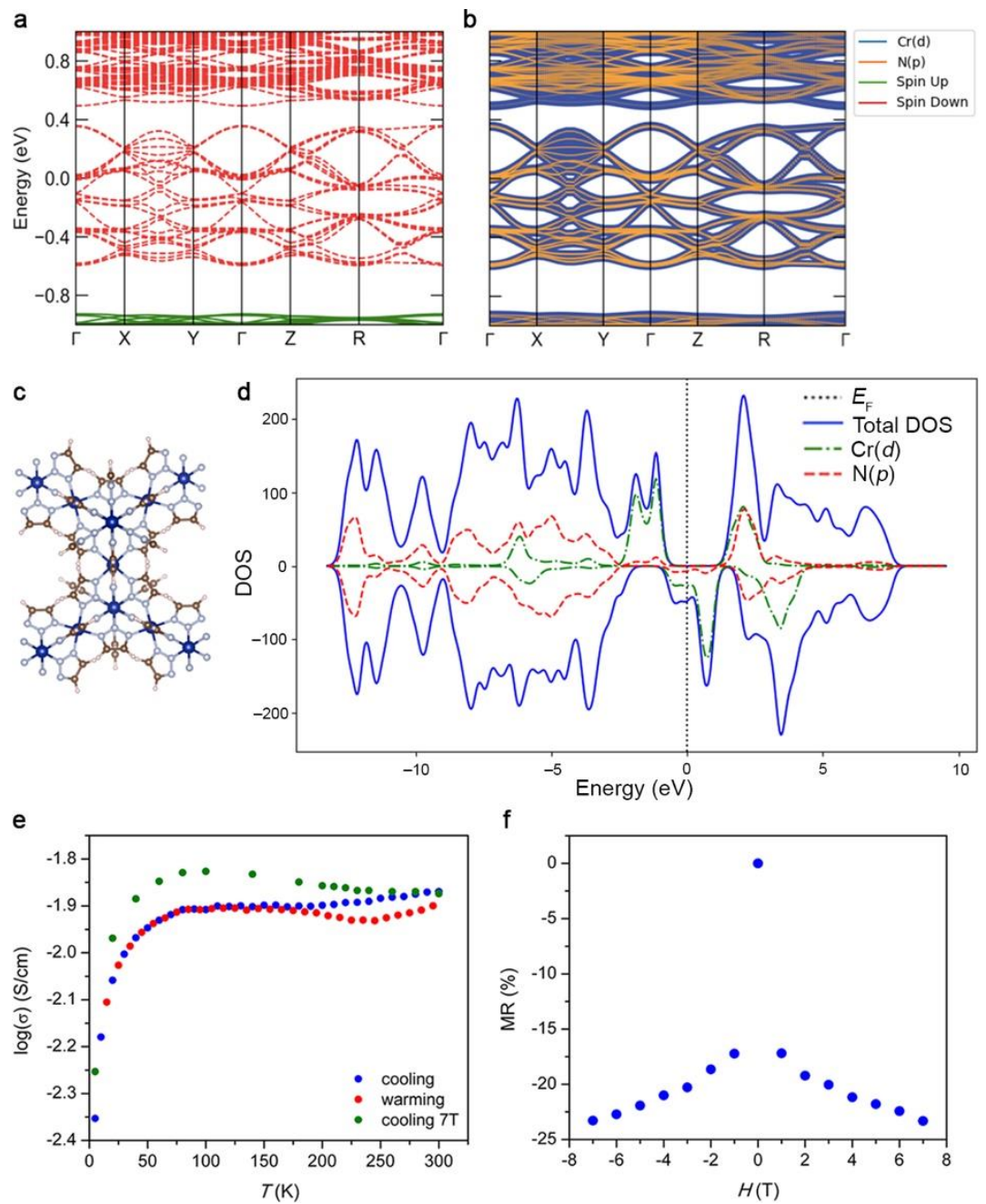

Figure 4 Electronic structure calculations and electronic conductivity of $\mathrm{Cr}(\operatorname{tri})_{2}\left(\mathrm{CF}_{3} \mathrm{SO}_{3}\right)_{0.33}$ $\mathbf{a , b}$, Total (a) and projected (b) band structure for the ferromagnetic state, calculated using the Perdew-Burke-Brnzerhof (PBE) functional. The zero of energy is set to the highest occupied state. The symmetry points correspond to $\Gamma=(0,0,0), X=(0.5,0,0), Y=(0,0.5,0), Z=(0,0,0.5)$, and $R$ $=(0.5,0.5,0.5)$. c, The geometry-optimized structure of $\mathrm{Cr}(\operatorname{tri})_{2}\left(\mathrm{CF}_{3} \mathrm{SO}_{3}\right)_{0.33}$. Blue, light blue, brown, and white spheres represent $\mathrm{Cr}, \mathrm{N}, \mathrm{C}$, and $\mathrm{H}$ atoms, respectively; $\mathrm{CF}_{3} \mathrm{SO}_{3}{ }^{-}$ion has been omitted for clarity. $\mathbf{d}$, Partial density of states (pDOS) in arbitrary units for the ferromagnetic state. The zero of energy is set to the highest occupied state denoted by the dotted vertical line. e, Variable-temperature conductivity data of $\mathrm{Cr}(\operatorname{tri})_{2}\left(\mathrm{CF}_{3} \mathrm{SO}_{3}\right)_{0.33}$. Blue and red spheres represent measurements taken during cooling and warming, respectively. Green spheres represent data collected while cooling under an applied magnetic field of $7 \mathrm{~T}$. f, Magnetoresistance data of $\mathrm{Cr}(\text { tri })_{2}\left(\mathrm{CF}_{3} \mathrm{SO}_{3}\right)_{0.33}$ collected at $5 \mathrm{~K}$ under selected dc magnetic fields. 


\section{Methods}

Materials. All manipulations were performed under an Ar atmosphere in an Mbraun glovebox or using standard Schlenk techniques. Anhydrous $N, N$-dimethylformamide (DMF) and dichloromethane were obtained from a JC Meyer solvent system and stored over 3 - or $4-\AA$ molecular sieves prior to use. The molecule $1 H-1,2,3$-triazole (98\% purity) was purchased from VWR International LLC, deoxygenated with three successive freeze-pump-thaw cycles, and dried over 4 - $\AA$ molecular sieves before use. Anhydrous $\mathrm{Cr}\left(\mathrm{CF}_{3} \mathrm{SO}_{3}\right)_{2}$ was prepared according to a previously reported procedure (ref. 36). Elemental analyses for $\mathrm{C}, \mathrm{H}$, and $\mathrm{N}$ were obtained from the Microanalytical Laboratory at the University of California, Berkeley.

Synthesis of $\mathbf{C r}(\mathbf{t r i})_{2}\left(\mathbf{C F}_{3} \mathrm{SO}_{3}\right)_{0.33}$. Liquid $1 \mathrm{H}$-1,2,3-triazole (1.04 g, $\left.15.0 \mathrm{mmol}\right)$ was added to a 20 -mL glass scintillation vial containing a solution of $\mathrm{Cr}\left(\mathrm{CF}_{3} \mathrm{SO}_{3}\right)_{2}(1.75 \mathrm{~g}, 5.00 \mathrm{mmol})$ in $10 \mathrm{~mL}$ of DMF. The vial was sealed with a polytetrafluoroethylene-lined cap and heated at $120{ }^{\circ} \mathrm{C}$ for 3 days. The resulting suspension was cooled to $25^{\circ} \mathrm{C}$ and filtered with a Nylon membrane filter to yield a dark purple powder. The powder was washed by soaking in DMF $(3 \times 15 \mathrm{~mL})$ and then in dichloromethane $(4 \times 15 \mathrm{~mL})$, and was dried under dynamic vacuum $(<10 \mu \mathrm{bar})$ at $130{ }^{\circ} \mathrm{C}$ for 48 $\mathrm{h}$ to afford $0.356 \mathrm{~g}(30 \%)$ of product as a purple microcrystalline powder. Anal. Calcd for $\mathrm{CrC}_{4.33} \mathrm{H}_{4} \mathrm{~N}_{6} \mathrm{FS}_{0.33} \mathrm{O}$ : C, 21.91; H, 1.70; N, 35.41. Found: C, 22.24; H, 1.44; N, 35.21.

Magnetic measurements. In a glovebox under an $\mathrm{N}_{2}$ atmosphere, a sample was prepared by adding an activated powder into a 5-mm-inner-diameter quartz tube containing a raised quartz platform. The sample powder was restrained with a plug of compacted glass wool to prevent crystallite torqueing during measurements. The quartz tube was transferred to a Schlenk line and evacuated until the internal pressure reached 30 mTorr. The tube was cooled in liquid $\mathrm{N}_{2}$ and flame-sealed under static vacuum. All magnetic measurements were performed using a Quantum Design MPMS2 SQUID magnetometer from 3 to $350 \mathrm{~K}$ at applied magnetic fields ranging from 0 to $\pm 7 \mathrm{~T}$. The ac susceptibility measurements were performed with an oscillating field of 4 Oe with a frequency from 1 to $100 \mathrm{~Hz}$. Diamagnetic corrections were applied to the data using Pascal's constants to give $\chi_{\mathrm{D}}=-0.00009744 \mathrm{emu} \mathrm{mol}^{-1}$.

Electrical conductivity measurements. Conductivity measurements were performed using a home-built four-contact cell (Extended Data Fig. 5a). In a glovebox under an Ar atmosphere, a sample pellet was manually pressed in a cell with a known inner cell diameter. While pressed, the cell was sealed with Torr Seal ${ }^{\circledR}$ low-vapor-pressure epoxy. When the epoxy dried completely, the cell was removed from the press for the sample pellet thickness to be measured using a caliper. Room temperature conductivity measurement was performed using a Bio-Logic VMP-3 multipotentiostat fitted to the Ar glovebox. Variable-temperature conductivity measurements were performed in a Quantum Design MPMS2 SQUID magnetometer with a modified sample rod that accommodates ten 26 AWG silver coated copper cables sealed at the top of the rod with an air tight Swagelock fitting and Torr Seal ${ }^{\circledR}$ low-vapor-pressure epoxy. The airtight sample cell was attached to the SQUID sample rod and inserted into the cryostat-equipped SQUID chamber at 300 $\mathrm{K}$. $I-V$ profiles between 5 and $300 \mathrm{~K}$ were collected with a Bio-Logic SP200 potentiostat by scanning current between $\pm 50 \mu \mathrm{A}$ and the sample cell allowed to equilibrate for at least $30 \mathrm{~min}$ 
between each measured temperature. All data collected were ohmic within a $\pm 50 \mu \mathrm{A}$ window and were then modelled with Ohm's Law, $E \times \sigma=j$, where $E$ is the applied electric field and $j$ is the current density, to determine the sample conductivity with $\sigma$ with the units of $\mathrm{S} \mathrm{cm}^{-1}$. Magnetoresistance measurements were performed by applying magnetic fields ranging from 0 to \pm 7 T during $I-V$ profile collections. Data were fitted to charge-transport models as described in the Supplementary Information.

Surface area measurement. Gas adsorption isotherms for pressures in the range of 0-1 bar were measured using a Micrometrics ASAP2020 gas adsorption analyser instrument. In an $\mathrm{N}_{2}$-filled glovebox, the samples were transferred to a pre-weighted analysis tube and capped with a Micrometrics TranSeal. The analysis tube containing the sample was transferred to a Micrometrics Smart VacPrep instrument and evacuated by heating to $130{ }^{\circ} \mathrm{C}$ with a ramp rate of $2{ }^{\circ} \mathrm{C} \min ^{-1}$ under dynamic vacuum for $48 \mathrm{~h}$, until an outgas rate of $<3 \mu \mathrm{bar} \mathrm{min}^{-1}$ was achieved. The evacuated tube was carefully transferred to an electronic balance and weighed to determine the sample mass. The analysis tube was then transferred to an analysis port of the Micrometrics ASAP2020 instrument. An $\mathrm{N}_{2}$ isotherm at $77 \mathrm{~K}$ was measured using a UHP-grade gas source. For the determination of surface areas, the Langmuir and BET methods were applied using Micrometrics software, assuming an $\mathrm{N}_{2}$ cross-sectional area of $16.2 \AA^{2}$ molecule $^{-1}$.

High-resolution powder $\mathbf{X}$-ray diffraction. The powder sample was packed into a 1.0-mm boron-rich glass capillary tube inside a glovebox under an $\mathrm{N}_{2}$ atmosphere. The capillary was flamesealed and placed inside a Kapton tube that was sealed on both ends with epoxy. High-resolution synchrotron X-ray powder diffraction data were collected at Beamline 11-BM at the Advanced Photon Source (APS) at Argonne National Laboratory. Diffraction patterns were collected at 360, 220 , and $120 \mathrm{~K}$ with a wavelength of $0.412685 \AA$. Discrete detectors covering an angular range from -6 to $16^{\circ}$ in $2 \theta$ were scanned over a $34^{\circ}$ range of $2 \theta$, with data points collected every $0.001^{\circ}$ in $2 \theta$ and at scan speed of $0.01^{\circ} \mathrm{s}^{-1}$. For all diffraction data from Beamline 11-BM, precise unitcell dimensions were determined by performing Pawley refinements, after which Rietveld refinements were performed using the software package TOPAS-Academic (ref. 37). Highresolution synchrotron X-ray powder diffraction data between 270 and 5 K (Extended Data Fig. 7a) were collected at Beamline BM31 at the European Synchrotron Radiation Facility (ESRF) with the wavelength of $0.49754758 \AA$.

Infrared spectroscopy. Infrared spectra were collected using a Bruker Vertex 70 spectrometer equipped with a glowbar source, $\mathrm{KBr}$ beamsplitter, and a liquid nitrogen cooled mercurycadmium-telluride detector. A custom-built diffuse reflectance system was used for all measurements. Sample temperature was controlled by an Oxford Instruments OptistatDry TLEX cryostat, and sample atmosphere was controlled by a Micromeritics ASAP 2020Plus gas sorption analyzer. In a typical experiment, material was dispersed in dry $\mathrm{KBr}(10 \mathrm{wt} \%)$ in an Ar-filled glovebox and evacuated under turbovac. Spectra were collected at various temperatures until no further changes were observed. 
UV-visible-NIR diffuse reflectance spectroscopy. UV-vis-NIR diffuse reflectance spectra were collected using a CARY 5000 spectrophotometer interfaced with Varian Win UV software. The sample was prepared in an Ar-filled glovebox and held in a Praying Mantis air-free diffuse reflectance cell. Barium sulfate powder was used as a non-abosrbing matrix. The spectrum was collected in $F(R)$ vs wavenumber, where $F(R)$ is the Kublelka-Munk conversion $F(R)=(1-R)^{2} / 2 R$ of the raw diffuse reflectance, $R$.

Scanning electron microscopy. The sample for scanning electron microscopy (SEM) imaging was prepared by dispersing a powder sample of $\mathrm{Cr}(\operatorname{tri})_{2}\left(\mathrm{CF}_{3} \mathrm{SO}_{3}\right)_{0.33}$ in dichloromethane followed by drop casting onto a silicon wafer. SEM images were obtained using a Hitachi S-5000 instrument at $10 \mathrm{keV}$ and $4 \mathrm{nA}$.

Powder neutron diffraction. Powder neutron diffraction data were collected on the POWGEN instrument at the Spallation Neutron Source (SNS) at Oak Ridge National Laboratory (ORNL). Approximately one gram of sample was loaded into a vanadium POWGEN Automatic Changer can in an He-filled glovebox. Data were collected at the selected temperatures of 300, 150, and 10 $\mathrm{K}$ in the high-resolution mode (ref. 38).

\section{Data availability}

Additional crystallographic information, powder X-ray diffraction data, scanning electron microscopy, gas-sorption data, electronic structure calculations, magnetic data, and conductivity data are available in the Supplementary Information and Extended Data.

36. Dixon, N. E., Lawrance, G. A., Lay, P. A., Sargeson, A. M. \& Taube, H. Trifluoromethanesulfonates and trifluoromethanesulfonato-o complexes. Inorg. Synth. 28, 70-76 (1990).

37. Coelho, A. A. TOPAS-Academic, version 5 (Coelho Software, 2017)

38. Huq, A. et al. POWGEN: rebuild of a third-generation powder diffractometer at the Spallation Neutron Source. J. Appl. Cryst. 52, 1199-1201 (2019).

Acknowledgements This research was supported by National Science Foundation (NSF) Award No. DMR-1611525, with the exception of the measurement and analysis of the magnetic data, which were supported by the Nanoporous Materials Genome Center of the U.S. Department of Energy, Office of Basic Energy Sciences, Division of Chemical Sciences, Geosciences and Biosciences under Award No. DE-FG02-17ER16362. Powder X-ray diffraction data were collected at Beamline 11-BM at the Advanced Photon Source, operated by Argonne National Laboratory, and beamline BM31 at the European Synchrotron Radiation Facility (ESRF), Grenoble, France. We are grateful to Local Contact at the ESRF for providing assistance in using beamline BM31. Use of the Advanced Photon Source at Argonne National Laboratory was supported by U.S. Department of Energy, Office of Science, Office of Basic Energy Sciences, under Contract No. DE-AC02-06CH11357. Data from Beamline 11-BM were collected as part of the 2018 Modern Methods in Rietveld Refinement and Structural Analysis workshop, a school supported by the US national Committee for Crystallography and the American Crystallographic Association. Neutron diffraction data were collected at the POWGEN beamline at the Spallation 
Neutron Source, a DOE Office of Science User Facility operated by the Oak Ridge National Laboratory. Electronic structure calculations utilized an award of computer time provided by ASCR Leadership Computing Challenge (ALCC) program and resources of the National Energy Research Scientific Computing Center (NERSC), a U.S. Department of Energy Office of Science User Facility operated under Contract No. DE-AC02-05CH11231. Additional computation resources were provided by the Minnesota Supercomputing Institute (MSI) at the University of Minnesota. T.R. thanks the Welch Foundation (Grant No. N-2012-20190330) for funding. In addition, we thank M. E. Ziebel, J. Oktawiec, S. H. Lapidus, X. Wenqian, P. Khalifah, Q. Zhang, and M. J. Kirkham for discussions and experimental assistance, and Prof. T. D. Harris for editorial assistance. We also thank the National Science Foundation and National GEM Consortium for providing graduate fellowship supports for J.G.P and B.A.C, respectively.

Author contributions J.G.P. and J.R.L. formulated the project. J.G.P. synthesized the compound. B.A.C. and J.D.G. performed electronic structure calculations and analyzed data. J.G.P. and L.E.D. collected and analyzed the magnetic data. J.G.P. and M.L.A. collected and analyzed conductivity data. E.V. collected SEM images. H.J. collected and analyzed the infrared spectra. J.G.P. collected and analyzed powder X-ray diffraction data from APS, with assistance from T.R. M.A.G. collected and analyzed powder X-ray diffraction data from ESRF. M.A.G. and J.G.P. collected and analyzed powder neutron diffraction data. J.G.P. and J.R.L. wrote the paper, and all authors contributed to revising it.

Competing interests The authors declare no competing interests.

\section{Additional information}

Supplementary information is available for this paper.

Correspondence and requests for materials should be addressed to J.R.L. 


\section{Extended Data}

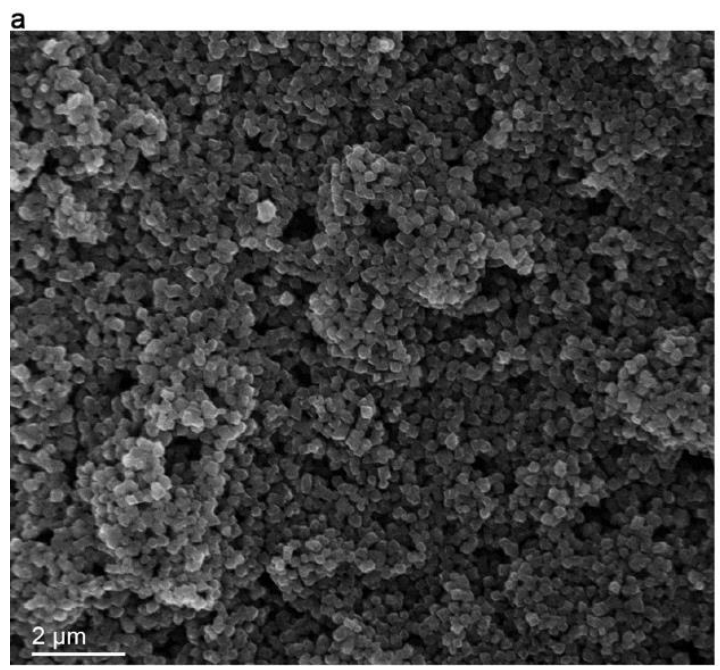

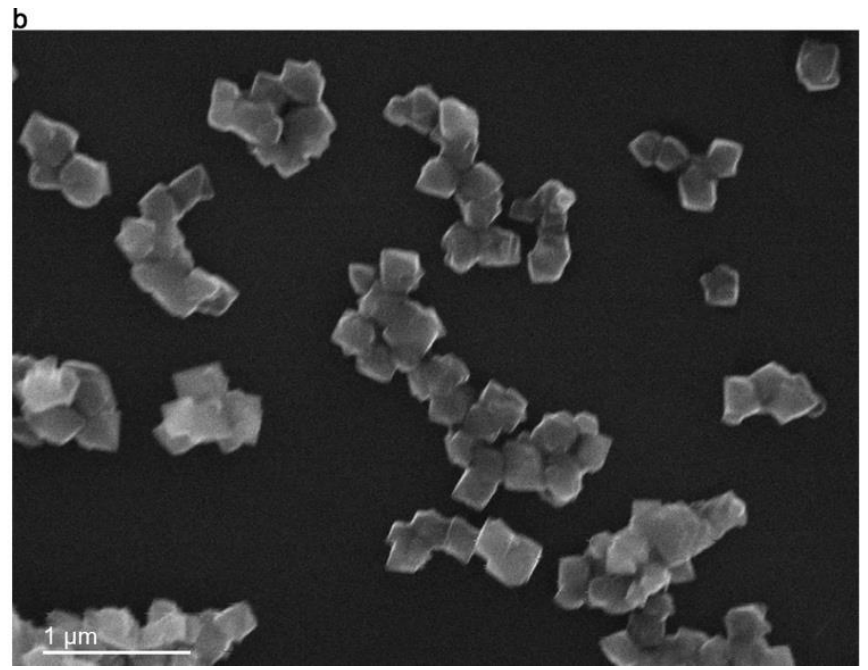

Extended Data Fig. 1 | Scanning electron microscopy images of $\mathrm{Cr}(\operatorname{tri})_{2}\left(\mathrm{CF}_{3} \mathrm{SO}_{3}\right)_{0.33}$ crystals. 

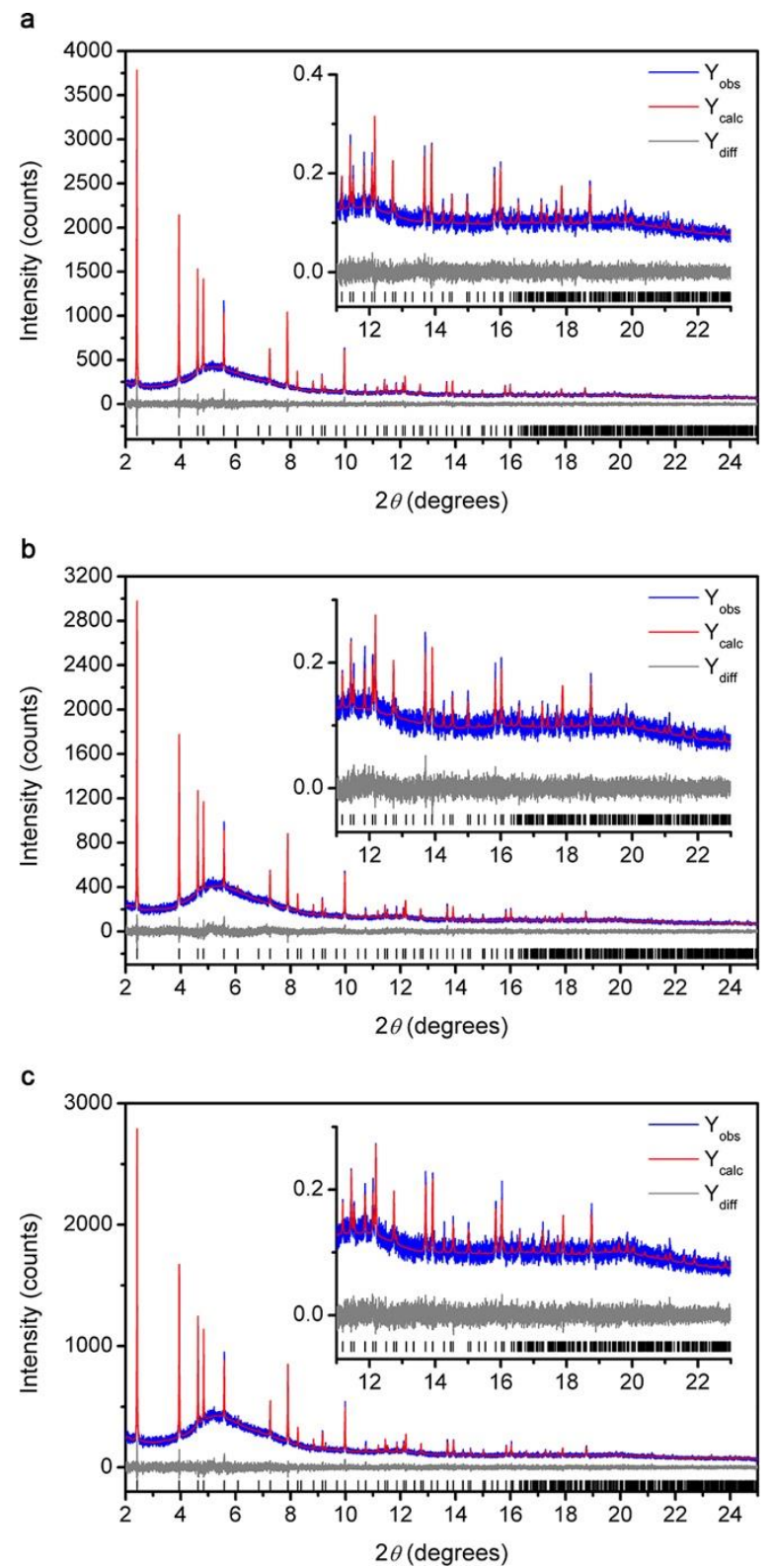

Extended Data Fig. 2 | Rietveld refinements. a-c, Rietveld refinements performed on the synchrotron powder X-ray diffraction patterns of $\mathrm{Cr}(\operatorname{tri})_{2}\left(\mathrm{CF}_{3} \mathrm{SO}_{3}\right)_{0.33}$ at (a) 360, (b) 220, and (c) $120 \mathrm{~K}$ from $2^{\circ}$ to $25^{\circ}$. Blue and red lines represent the observed and calculated diffraction patterns, respectively. The gray line represents the difference between observed and calculated patterns, and the black tick marks indicate calculated Bragg peak positions. The inset shows the high angle region at a magnified scale. The wavelength was $0.412685 \AA$. Figure-of-merit (as defined by TOPAS): (a) $R_{\mathrm{wp}}=6.76 \%, R_{\mathrm{p}}=5.20 \%, R_{\mathrm{bragg}}=1.73 \%, R_{\mathrm{wp}(\text { Rietveld })} / R_{\mathrm{wp}(\text { Pawley })}=1.01 ;(\mathbf{b}) R_{\mathrm{wp}}=$ $6.97 \%, R_{\mathrm{p}}=5.40 \%, R_{\mathrm{bragg}}=1.78 \%, R_{\mathrm{wp}(\text { Rietveld })} / R_{\mathrm{wp}(\text { Pawley })}=1.04 ;(\mathbf{c}) R_{\mathrm{wp}}=6.76 \%, R_{\mathrm{p}}=5.20 \%$, $R_{\text {bragg }}=1.75 \%, R_{\mathrm{wp}(\text { Rietveld })} / R_{\mathrm{wp}(\text { Pawley })}=1.00$. 

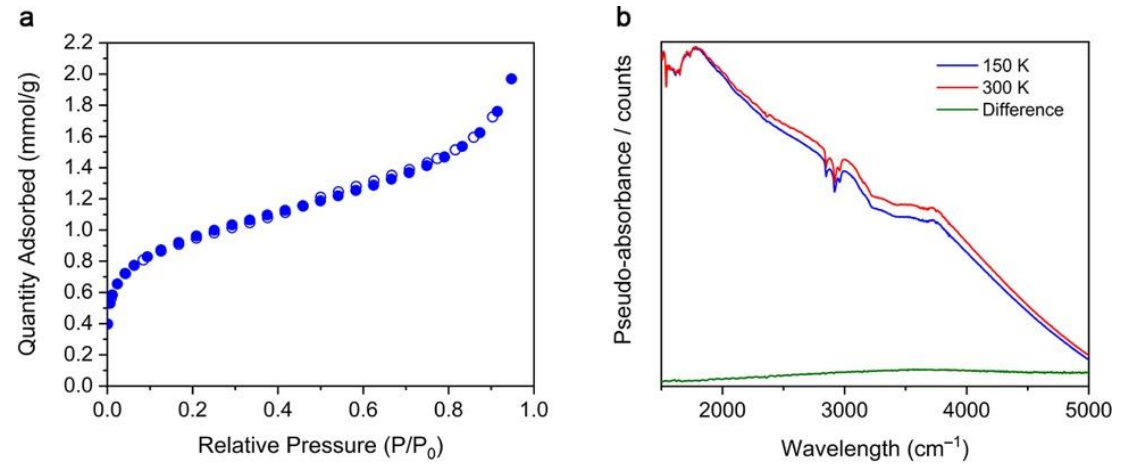

Extended Data Fig. 3 | Analyses of the $\mathrm{Cr}$ mixed-valency. a, $\mathrm{N}_{2}$ adsorption isotherm collected at $77 \mathrm{~K}$ for $\mathrm{Cr}(\operatorname{tri})_{2}\left(\mathrm{CF}_{3} \mathrm{SO}_{3}\right)_{0.33}$. Closed and open data points represent adsorption and desorption, respectively. b, Infrared data collected at $150 \mathrm{~K}$ (blue) and $300 \mathrm{~K}$ (red) and the difference plot (green) for $\mathrm{Cr}(\operatorname{tri})_{2}\left(\mathrm{CF}_{3} \mathrm{SO}_{3}\right)_{0.33}$. 


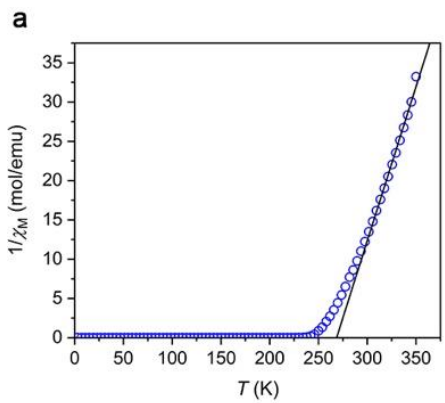

d

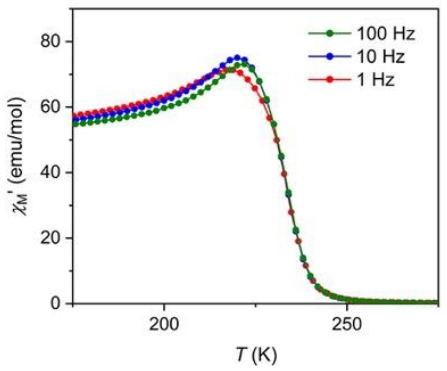

b

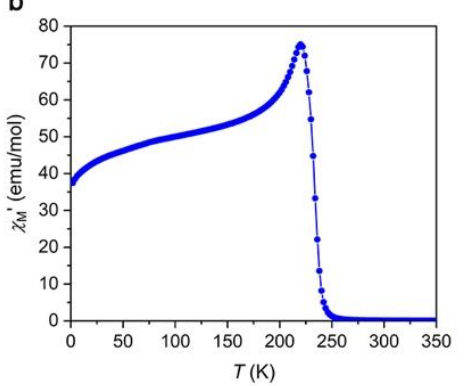

e

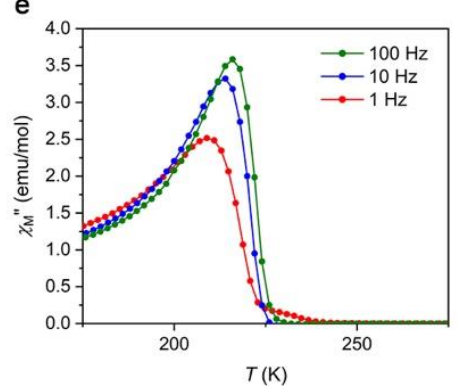

C

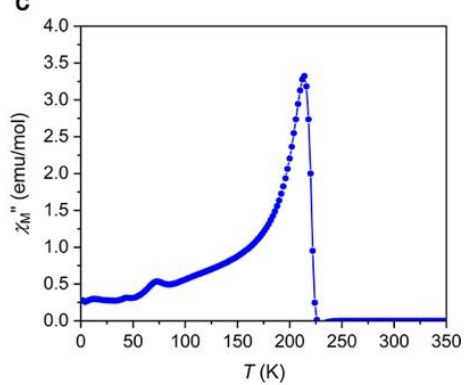

f

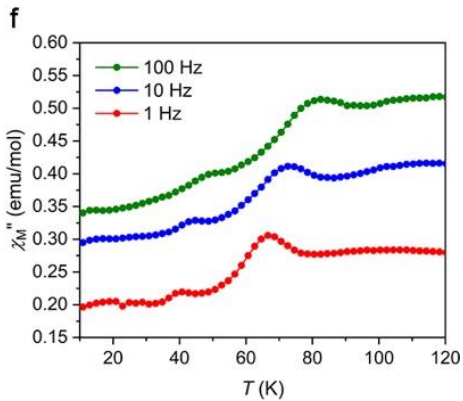

Extended Data Fig. 4 | Magnetic data. a, Variable-temperature magnetic susceptibility data $\left(\chi_{\mathrm{M}}\right)$ for $\mathrm{Cr}(\mathrm{tri})_{2}\left(\mathrm{CF}_{3} \mathrm{SO}_{3}\right)_{0.33}$ collected under the applied dc field of $25 \mathrm{Oe}$. Data plotted as $1 / \chi_{\mathrm{M}}$ versus temperature. Curie-Weiss fit to the data between $300 \mathrm{~K}$ and $350 \mathrm{~K}$ is shown by a black solid line, with fitting parameters described in the main text. b-f, Variable-temperature in-phase $\left(\chi^{\prime \prime}\right)$ and outof-phase $\left(\chi^{\prime \prime}\right)$ ac magnetic susceptibility of $\mathrm{Cr}(\operatorname{tri})_{2}\left(\mathrm{CF}_{3} \mathrm{SO}_{3}\right)_{0.33}$. at selected frequencies of $4 \mathrm{Oe}$ ac oscillating magnetic field and zero dc magnetic field. 

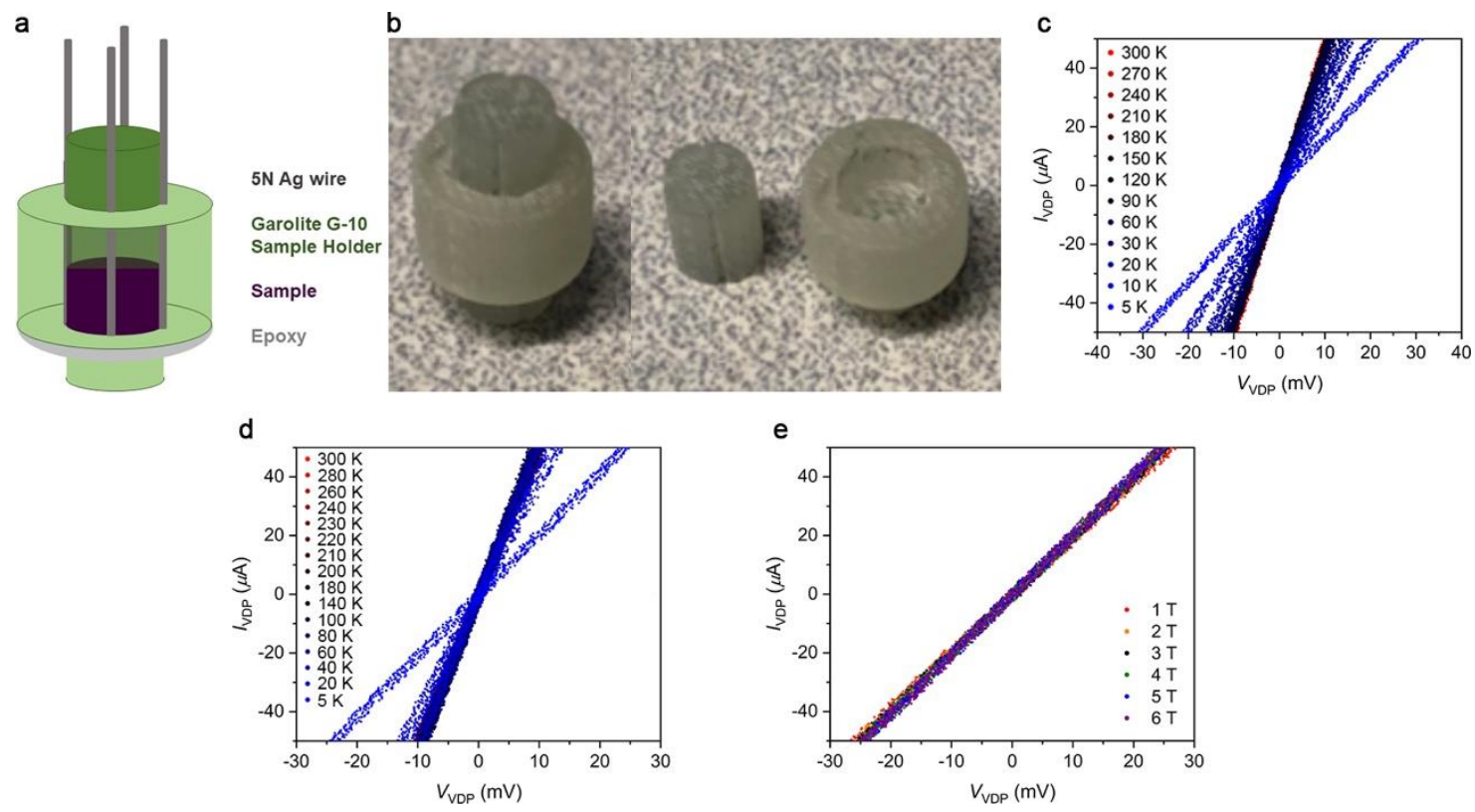

Extended Data Fig. 5 | Variable-temperature conductivity. a, b, Laboratory-constructed fourcontact conductivity measurement apparatus. c, Variable-temperature $I-V$ plots measured under zero applied magnetic field. d, Variable-temperature $I-V$ plots measured under applied magnetic field of $7 \mathrm{~T}$. e, $I-V$ plots measured at $5 \mathrm{~K}$ under selected applied magnetic fields. All $I-V$ profiles exhibited Ohmic response between $\pm 50 \mu \mathrm{A}$. 

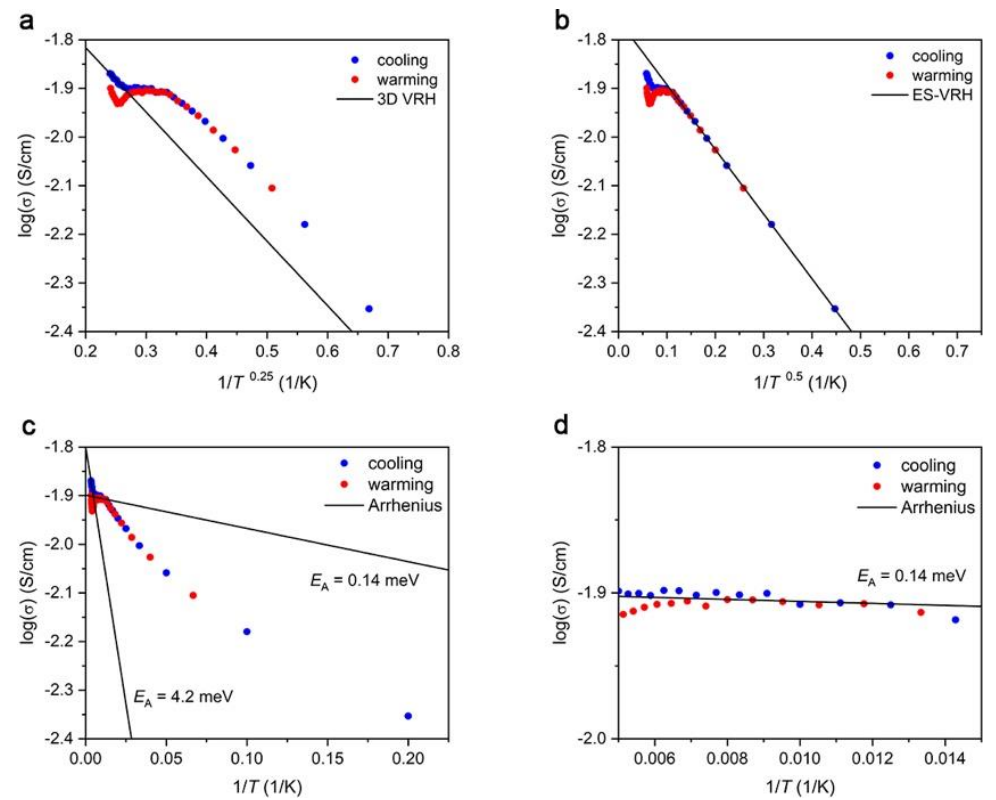

Extended Data Fig. 6 | Variable temperature conductivity data modeling. a-d, Variabletemperature $\log (\sigma)$ data of $\mathrm{Cr}(\operatorname{tri})_{2}\left(\mathrm{CF}_{3} \mathrm{SO}_{3}\right)_{0.33}$ plotted versus (a) $1 / T^{0.25}$, (b) $1 / T^{0.5}$, and (c, d) $1 / T$ and fitted with three-dimensional variable-range hopping, Efros-Shklovskii variable-range hopping, and Arrhenius models, respectively. For clarity, $\log (\sigma)$ versus $1 / T$ plot (c) has been zoomed-in to the intermediate temperature region (d) where conductivity exhibits a weak temperature dependence. 

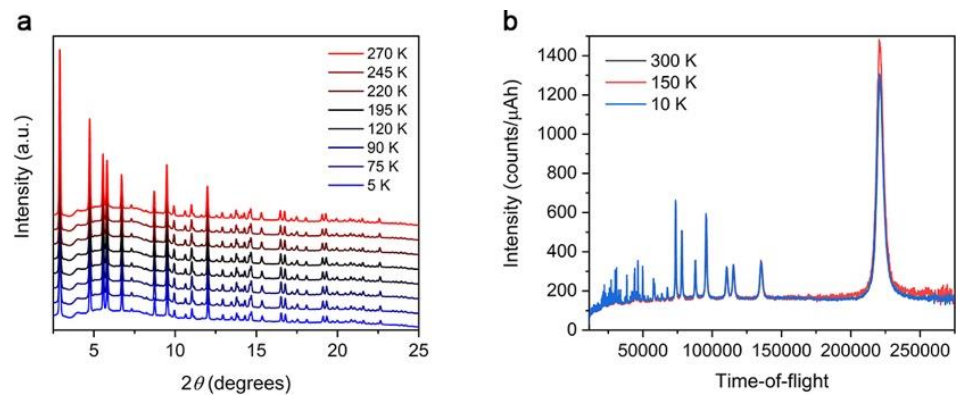

Extended Data Fig. 7 | Further diffraction studies. a, Variable-temperature synchrotron powder $\mathrm{X}$-ray diffraction patterns of $\mathrm{Cr}(\mathrm{tri})_{2}\left(\mathrm{CF}_{3} \mathrm{SO}_{3}\right)_{0.33}$ collected at selected temperatures between 5 and $270 \mathrm{~K}$. The wavelength was $0.49754758 \AA$ A. b, Powder neutron diffraction data collected at 10,150 , and $300 \mathrm{~K}$. To clarify, 10 and $300 \mathrm{~K}$ diffraction patterns nearly overlap with similar peak intensities and positions. 

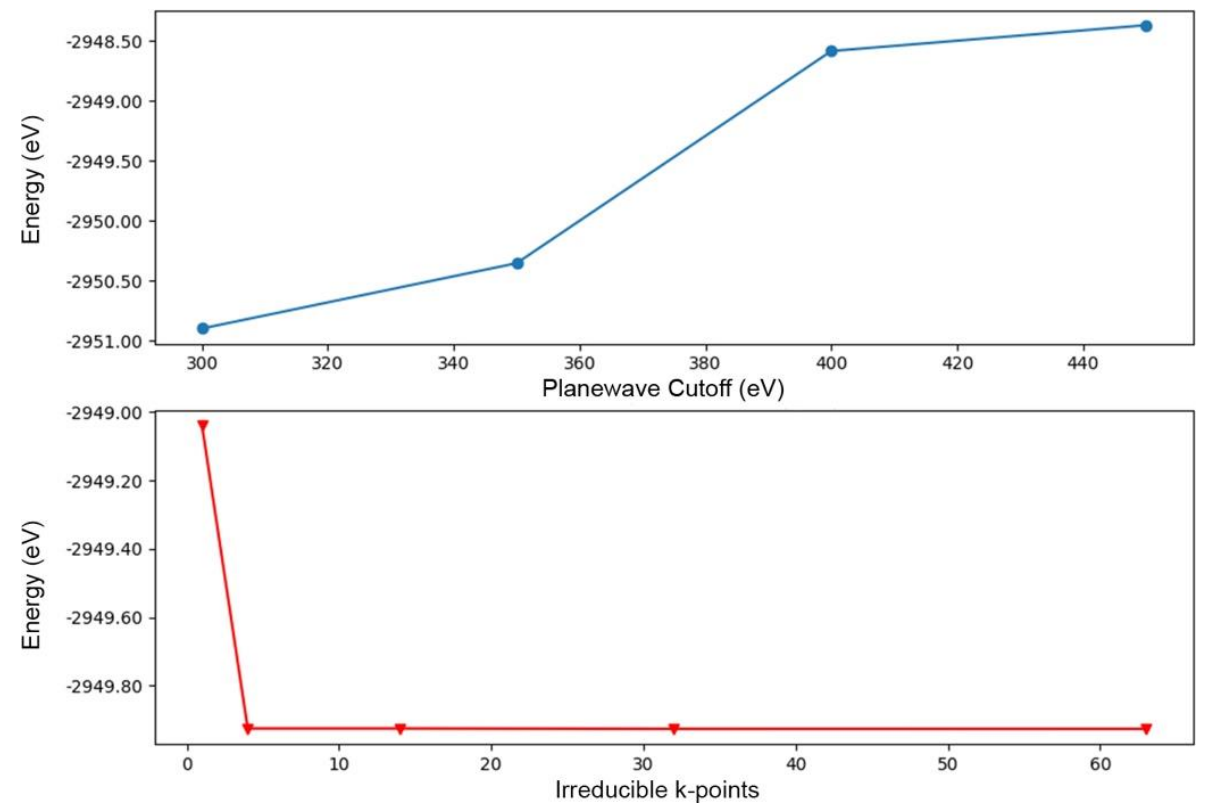

Extended Data Fig. 8 | Energy calculations. The energy of the optimized system is shown with respect to planewave cutoff and number of irreducible k-points. The calculations done varying planewave cutoff include 14 irreducible k-points. 
a

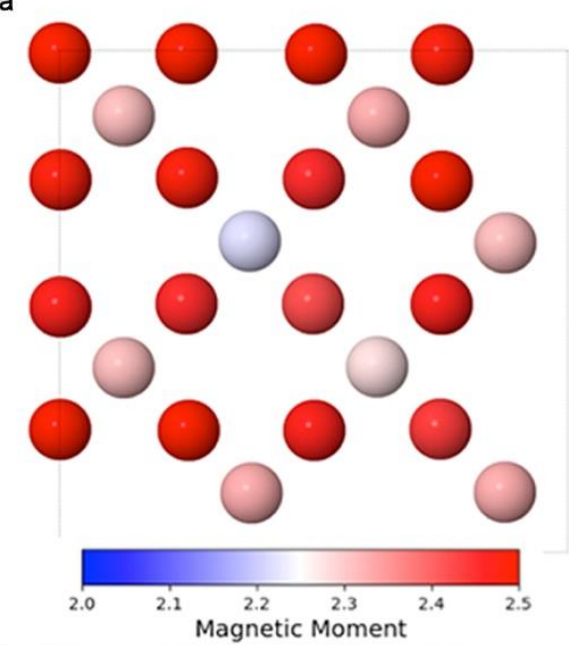

c

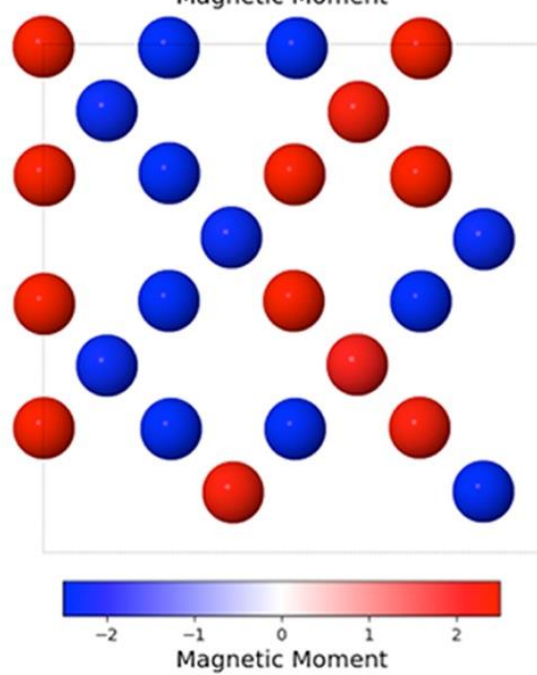

b

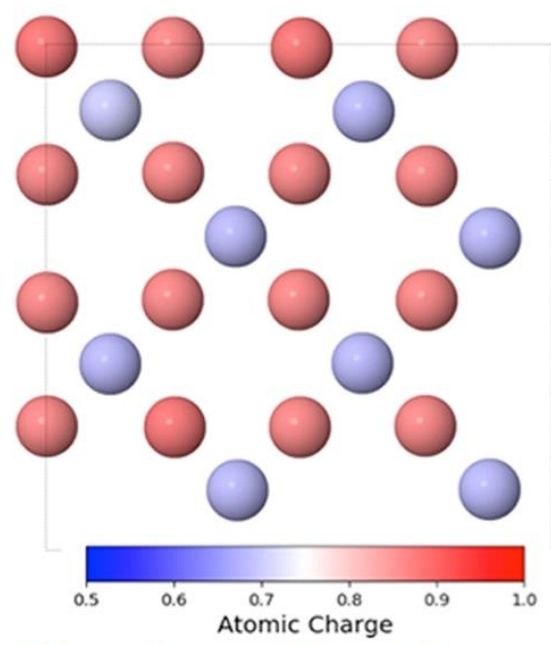

d

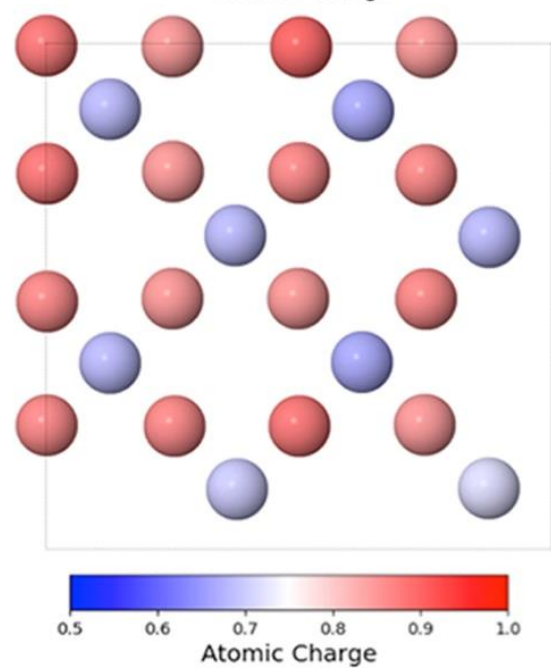

Extended Data Fig. 9 | Charge analysis. a, Magnetic moments $\left(\mu_{\mathrm{B}} \mathrm{mol}^{-1}\right)$ for the ferromagnetic state. $\mathbf{b}$, Atomic charge for the ferromagnetic state in atomic units. $\mathbf{d}$, Magnetic moments $\left(\mu_{\mathrm{B}} \mathrm{mol}^{-1}\right)$ for the antiferromagnetic state. $\mathbf{d}$, Atomic charge for the antiferromagnetic state in atomic units. Atomic properties shown as a gradient from blue to white to red. Negative magnetic moments indicate spin-down contributions. 


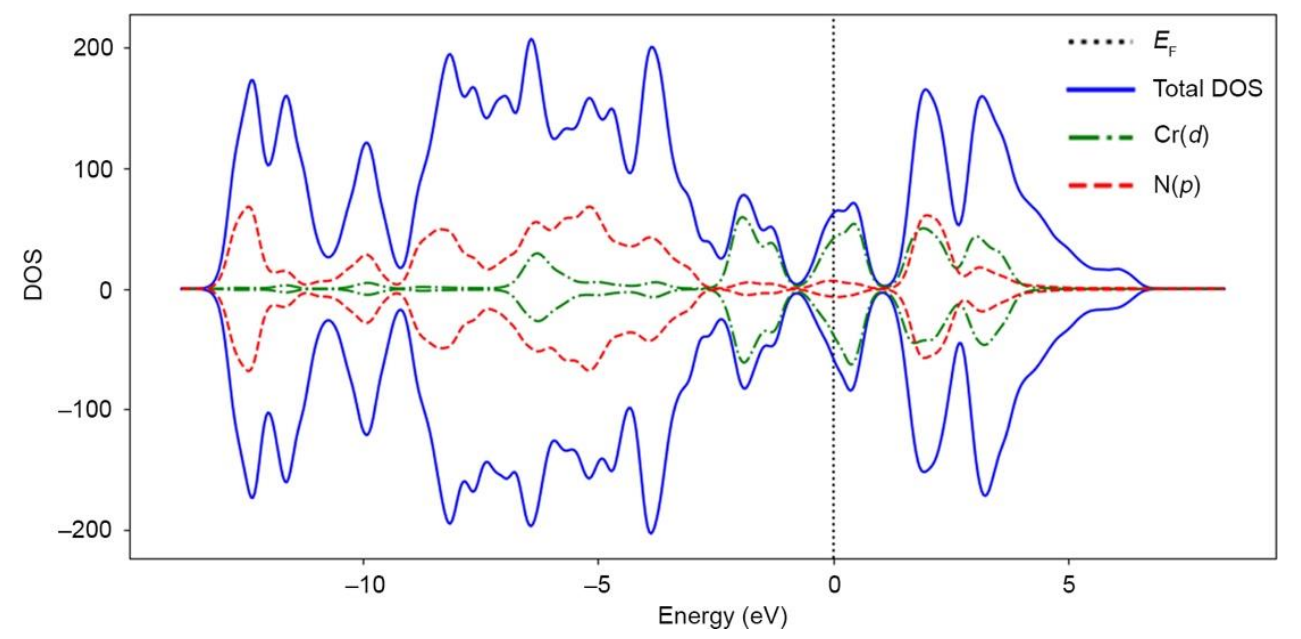

Extended Data Fig. 10 | Partial density of states (pDOS) of the antiferromagnetic state. Partial density of states (pDOS) in arbitrary units for the antiferromagnetic system. Blue, green, and red denote the total, $\mathrm{Cr} d$, and $\mathrm{N} p$, respectively. The zero of energy is set to the highest occupied state denoted by the dotted vertical line which has an energy of $2.60 \mathrm{eV}$. 